\title{
Design of an Ultra-Lightweight Autonomous Solar Airplane for Continuous Flight
}

\author{
André Noth ${ }^{1}$, Walter Engel and Roland Siegwart ${ }^{2}$ \\ 1 Autonomous Systems Lab, EPFL andre.noth@epfl.ch \\ 2 Autonomous Systems Lab, EPFL roland.siegwart@epfl.ch
}

Summary. The Autonomous Systems Lab of $\mathrm{EPFL}^{3}$ is developing, within the framework of an ESA program, an ultra-lightweight solar autonomous model airplane called Sky-Sailor with embedded navigation and control systems. The main goal of this project is to jointly undertake research on navigation, control of the plane and also work on the design of the structure, the energy generation system. The airplane will be capable of continuous flight over days and nights, which makes it suitable for a wide range of applications.

Key words: Autonomous UAV, solar powered airplane, sustainable flight

\section{Introduction}

Development of unmanned aerial vehicle (UAV) has attracted the attention of several agencies and university laboratories over the past decade, due to their great potential in military and civilian applications.

There are a dozen commercial autopilots (Micropilot, Procerus, etc.) which combine tiny dimensions, low weight and quite efficient navigation capabilities. Despite all this, they usually use limited CPU power which restricts the control of the airplane to classic control methods like separated PID loops and doesn't allow the onboard execution of more complex algorithms, for example, those of image processing.

On the other side, there is a lot of research in Universities in various fields, such as SLAM ${ }^{4}$, hardware design, control, navigation, trajectory planning, etc. But whether they are done on $\mathrm{VTOL}^{5}$ systems or fixed-wing model airplanes, the embedded system is often over-dimensioned, compared to the airplane itself, in order to have high computational capabilities and efficient sensors.

\footnotetext{
${ }^{3}$ Ecole Polytechnique Federale de Lausanne

${ }^{4}$ Simultaneous Localization and Mapping

${ }^{5}$ Vertical Take-Off and Landing
} 
Consequently, the UAV becomes very heavy, needs high electrical power and the flight endurance reduces dramatically. Thus, endurance being one of the most important parameters for the targeted applications, the development and the application are not in correlation.

In this paper, we present the airplane developed for the project Sky-Sailor whose aim is to build a solar autonomous motor glider by taking care of all aspects, not only the autopilot system but as well the mechanical structure, the solar generator, the energy storage, etc. It differs from other similar projects like Helios or Centurion by its low weight and low cost. The final airplane only weighs $2.5 \mathrm{~kg}$ and according to the AUVS-international is part of the High Altitude Long Endurance UAV category [3].

\section{Airplane Overview}

\subsection{Mechanical Structure}

The approach we chose for the design of the airplane was to combine the knowledge of aerodynamics engineers and the experience of lightweight model airplanes designers. The starting point for this design was the model airplane of Walter Engel that holds the world record for flight duration of over 15 hours with $1 \mathrm{~kg}$ of battery. Sky-Sailor version 1 is basically a motor-glider with a structural weight of only $0.6 \mathrm{~kg}$ for a wingspan of $3.2 \mathrm{~m}$ and a wing surface of $0.776 \mathrm{~m}^{2}$ (Fig. 1). The resulting total weight including motors, propeller, solar cells, batteries and controller is around $2.5 \mathrm{~kg}$.

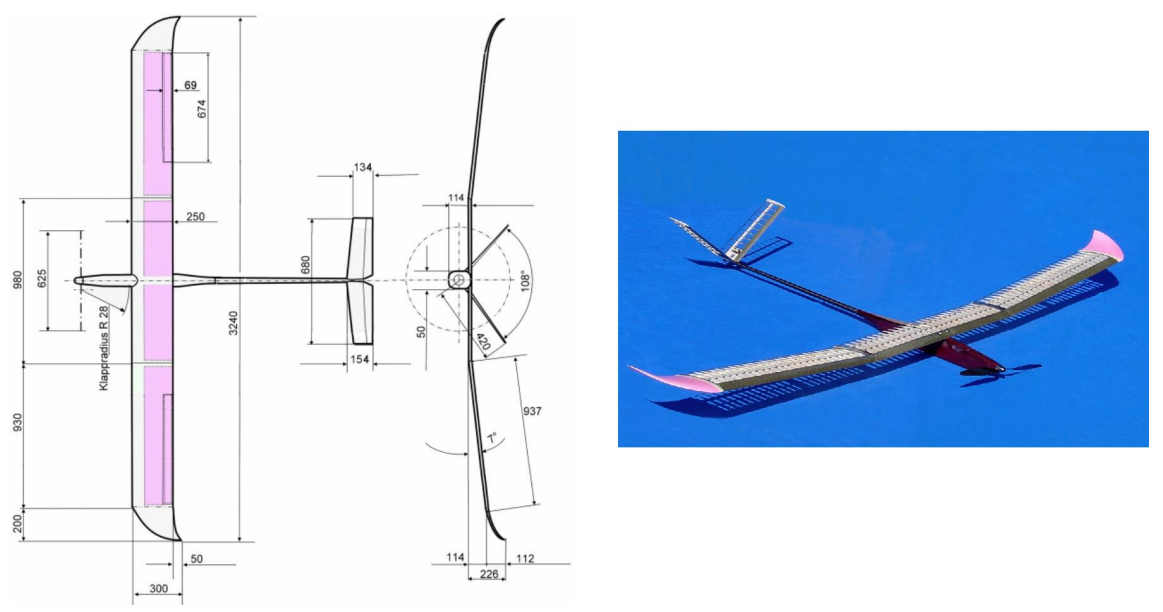

Fig. 1. Mechanical structure of Sky-Sailor 


\subsection{Solar generator, Battery and Propulsion System}

As explained in the introduction, one major challenge is the power management that has to ensure continuous flight over days and nights.

A total of 216 silicon solar cells, divided in three modules, cover an area of around $0.512 \mathrm{~m}^{2}$. In terms of efficiency, the better choice would have led us to GaAs Triple Junction cells with efficiencies of $27-28 \%$, but taking into account the impact of the weight on the required power for levelled flight, the better choice is RWE-32 silicon cells with $16.9 \%$ efficiency. Furthermore, the flexibility of those thin cells is also an advantage for their integration on the wing.

The cells are encapsulated using a mechanically favorable symmetrical laminate combined with a fiber glass reinforced plastic coating. This encapsulation is non-reflective. Thus, we obtain a flexible arrangement easily integrable on the plane and connectable to the power circuit. At maximum sun conditions, the available power is $28 \mathrm{~W}$ for each module, which makes a total of $84 \mathrm{~W}$.
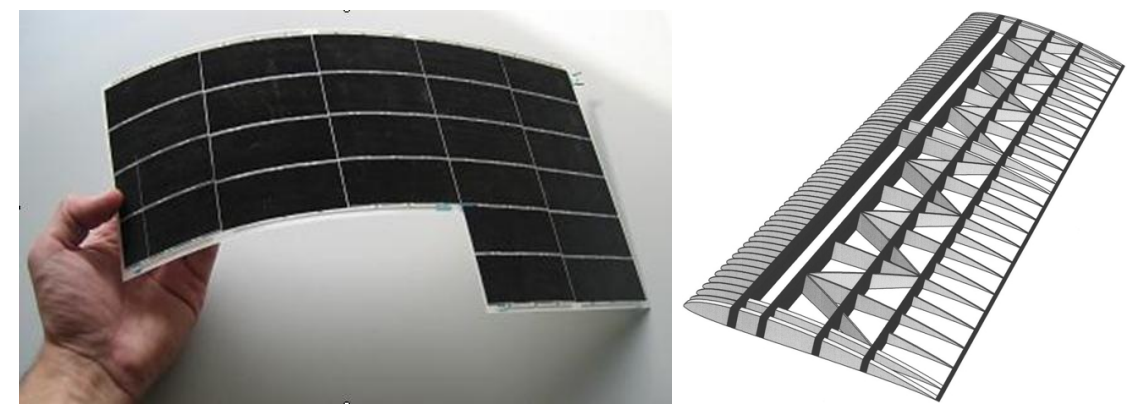

Fig. 2. Flexible solar module that can be directly integrated on the wing.

In order to get the highest amount of energy from the solar modules, a $\mathrm{MPPT}^{6}$ is used to charge the battery. This device is basically a high efficiency $\mathrm{DC} / \mathrm{DC}$ converter with variable and adjustable gain. One of its additional function is to monitor the current and the voltage of each solar module and make those information available for the central processor through $\mathrm{I}^{2} \mathrm{C}$.

The energy is stored in a lithium-ion polymer battery that has a nominal voltage of $28,8 \mathrm{~V}$ and a capacity of $7200 \mathrm{mAh}$. The propulsion group is composed of a Maxon DC motor, a gearbox and a carbon fiber propeller. The required electrical power for levelled flight of Sky-Sailor is around $16 \mathrm{~W}$.

\footnotetext{
${ }^{6}$ Maximum Power Point Tracker
} 


\section{Navigation and Control System}

In order to reach the goal of the project, the autopilot design phase followed those principles:

- select components not only according to criteria of precision and resolution, but as well of weight and power consumption to be suitable for the targeted application.

- use as much as possible digital output and calibrated sensor to reduce the development time and avoid additional need of $\mathrm{A} / \mathrm{D}$ converter, interface microcontroller, etc.

- interface the sensors so that the central processor doesn't have to wait on them but can access directly and rapidly to the information on request. This applies for example to the GPS.

\subsection{Computer and Interfaces}

Sky-Sailor will fly autonomously using an onboard autopilot, only high level orders being given from the ground. The system is mainly based on a single board computer, the X-board $<861>$ which is a compact embedded PC design for low power consumption.

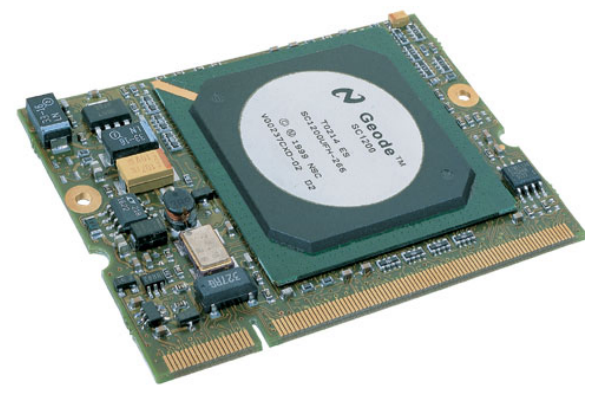

Fig. 3. X-board $<861>$ single board computer from Kontron

It includes a Geode SC1200 Processor, up to 128 Mbyte of DRAM and up to 128 Flash storage media on board. Despite the compact size of a business card, it offers a lot of interfaces: integrated Graphics, Ethernet, USB, RS 232, $\mathrm{I}^{2} \mathrm{C}$, audio... The OS running on it is a reduced Linux distribution, based on Debian, that only contains the necessary features.

\subsection{Sensors}

In Fig. 4, one can see the power generator system and the autopilot, with all sensors and their interfaces to the X-board. 


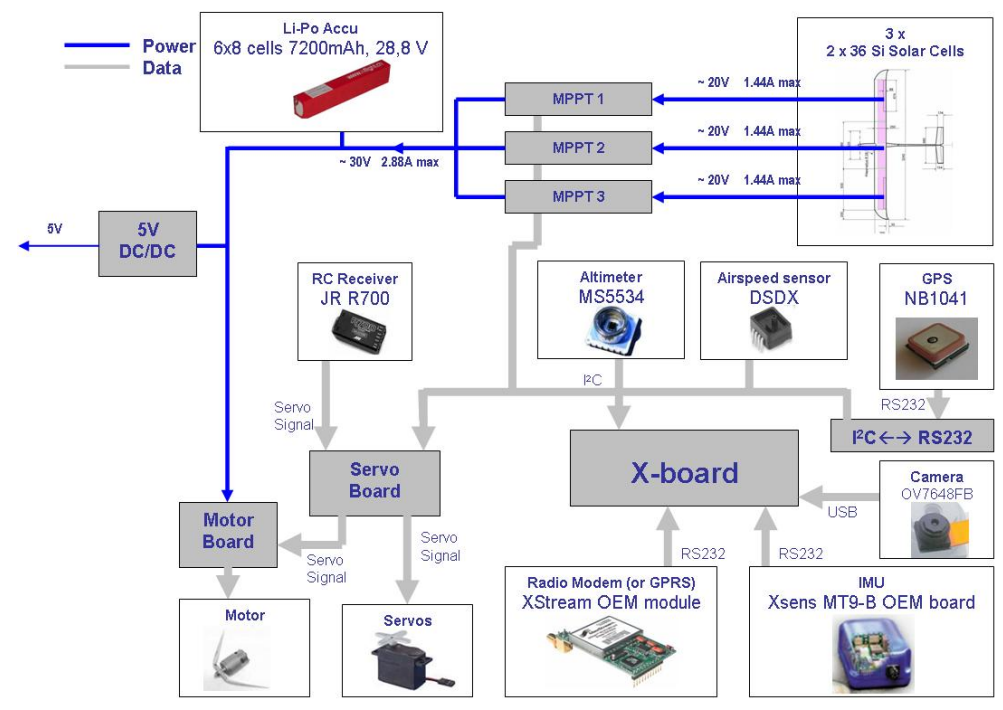

Fig. 4. Schematic view of the power and control parts of Sky-Sailor

\section{Attitude}

The attitude and angle rate of the airplane are given by the MT9-B IMU 7 at a frequency of up to $512 \mathrm{~Hz}$. Such a low-cost sensor is perfectly sufficient to perform inertial navigation compared to heavier one [6]. It contains accelerometers, magnetometers, gyroscopes and communicates through serial port (RS232) with the X-board on which data fusion is executed. In the future version of this device, the sensor fusion will be done by a DSP chip, reducing the computational cost on the central processor of the autopilot.

\section{VGA camera}

One direction of the project is to achieve autonomous navigation based on vision, using SLAM techniques as shown in [1] [2]. One or more lightweight VGA cameras will give $640 \times 480$ images of the landscape and allow localization and mapping of the terrain. Efforts will be done in this direction in the following month. Cameras are connected to the central processor via USB.

\section{Absolute $x-y$ position and altitude}

The absolute position is given by an ultra low power GPS sensor with patch antenna from Nemerix. This sensor consumes only $61 \mathrm{~mW}$ for a weight of 12.36 gr. In terms of position accuracy, $95 \% / 99.7 \%$ of the time, the estimated position lies within $2.7933 \mathrm{~m} / 4.2028 \mathrm{~m}$ respectively of the actual position.

\footnotetext{
${ }^{7}$ Inertial Measurement Unit
} 
A future version will accept WAAS/EGNOS correction for more precise measurements. The data are sent on a serial port at a fixed rate of $1 \mathrm{~Hz}$ to a microcontroller that decodes the NMEA protocol, stores the value internally and sends them on demand to the main processor via $\mathrm{I}^{2} \mathrm{C}$.

The same microcontroller interfaces the altitude pressure sensor MS5534. Pressure and temperature values, as well as four calibration factors allow the computation of the altitude with a resolution of $1 \mathrm{~m}$. The relation between pressure and altitude being variant with the atmospheric condition, the microcontroller will achieve data fusion, using the GPS altitude as an absolute value to correct the drift of the MS5534.

\section{Airspeed}

The airspeed sensor DSDX is a differential pressure sensor, with digital $\mathrm{I}^{2} \mathrm{C}$ readout and temperature compensated. It is connected to a Pitot tube fixed at the attack border of the wing.

\subsection{Ground Control Station}

The control of the airplane is executed onboard but there is a link to a ground control station through a serial radio modem that allows a baudrate of 9600 bps. The goal is to:

- download and upload airplane and control parameters, but as well the flight plan, before the takeoff,

- get a visual feedback of the state of the airplane once airborne, modify flight plans on-the-fly,

- retrieve and record the telemetry for flight analysis, system identification, etc.
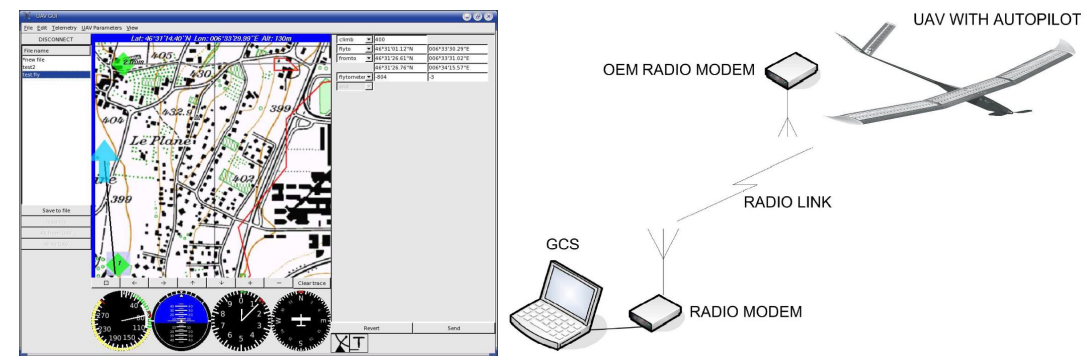

Fig. 5. Ground control station and it's graphical user interface

The GUI ${ }^{8}$ was developed with QT graphical libraries under Linux (Fig.5). It is composed of three main layers which ensures modularity:

\footnotetext{
${ }^{8}$ Graphical User Interface
} 
- the graphical interface, that allows a visual overview of the state of the airplane and its position on a 3D map of the terrain.

- a second layer which processes data and control the GUI

- a communication module that receives and sends the data in packets to the airplane through the serial port connected to the radio modem.

Control of the airplane from the ground

As shown in Fig. 4, the commands given to the servos can come from the autopilot or a human pilot on the ground using an RC transmitter. The "servo board" decodes the $\mathrm{PPM}^{9}$ from the $\mathrm{RC}$ source and get the value given by the autopilot through the $\mathrm{I}^{2} \mathrm{C}$ bus. Based on one additional channel on the $\mathrm{RC}$ remote, it switches from one source to the other. It is also possible, for control tuning purpose, to mix sources and, for example, allow the autopilot to command only the elevator while the other actuators are commanded manually.

\subsection{Autopilot Design Results}

The final design leads us to a navigation and control system with a total mass of $140 \mathrm{~g}$ for a consumption of around $4 \mathrm{~W}$. One can see that $6 / 8$ of the power is used by the $\mathrm{X}$-board and $1 / 8$ for the transmission, the rest being used by the sensors.

Table 1. Autopilot power and mass distribution

\begin{tabular}{|c|c|c|}
\hline Part & Weight $[\mathrm{g}]$ & Power consumption $[\mathrm{W}]$ \\
\hline X-board & 22 & 3.00 \\
\hline Mother Board & 22 & - \\
\hline IMU & 14.5 & 0.21 \\
\hline VGA Camera & 0.55 & 0.1 \\
\hline GPS & 12.4 & 0.061 \\
\hline Altitude sensor board & 2 & 0.03 \\
\hline Airspeed sensor board & 3 & 0.03 \\
\hline Radio-modem & 24 & 0.5 \\
\hline Antenna & 19.6 & - \\
\hline Cables, connectors & 20 & - \\
\hline Total & $140 \mathrm{~g}$ & $3.93 \mathrm{~W}$ \\
\hline
\end{tabular}

Globally, the autopilot represents $5 \%$ of the total mass of the airplane and uses $20 \%$ of the power.

\footnotetext{
${ }^{9}$ Pulse Period Modulation
} 


\section{Simulation of the solar flight}

For the validation of a long endurance solar flight, a simulation was realized under Matlab Simulink. Fig.6 represents the schematic of the model that includes first the irradiance model based on [12] and depending on the geographic position, time and solar panels orientation. We then take into account the surface of solar cells, their electrical efficiency and the efficiency of the connection configuration. For the MPPT, the electrical and algorithm efficiencies are taken into account. The power consumption is the addition of the autopilot power and the power needed for flight, which was measured in the case of levelled flight and climbing phase. Depending on the irradiance conditions and the consumption, the battery is charged or discharged, taking into account the efficiency of the energy transfer.

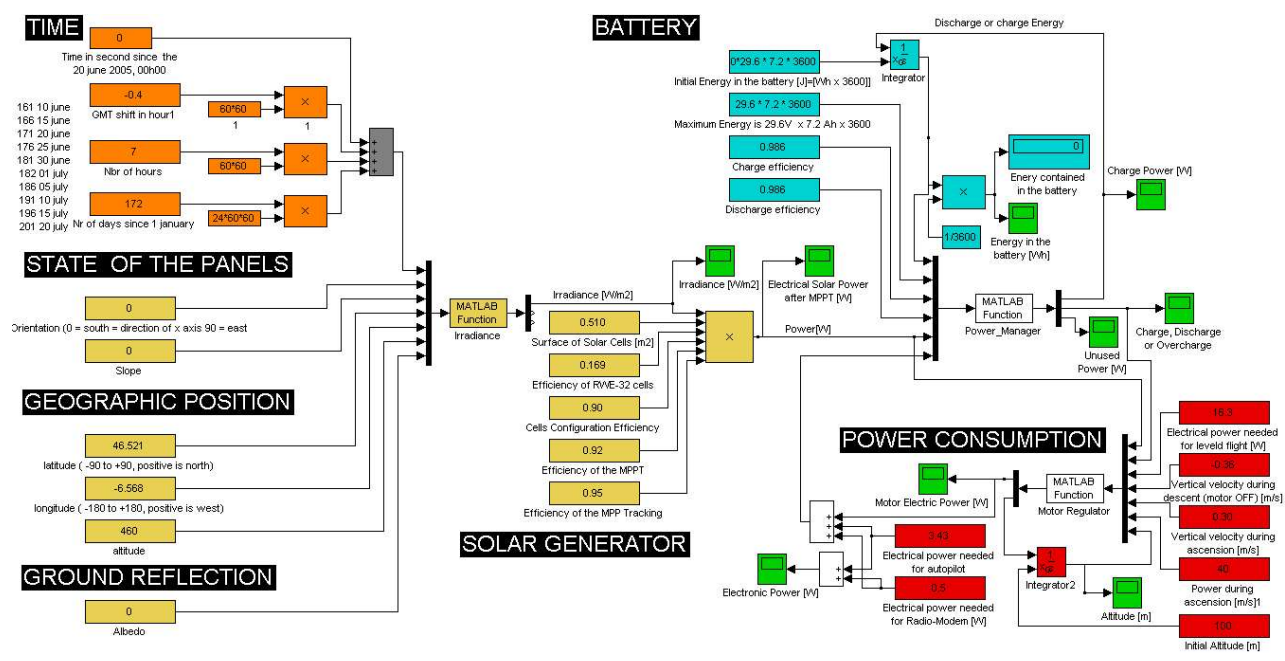

Fig. 6. Schematic of the simulation model under Matlab Simulink

\subsection{Study of various scenarios}

The simulation environment allows to test different flight strategies in order to accomplish a long endurance flight and analyze the benefit of a climbing phase or the influence of the other parameters on the feasibility of a multi-days flight. We will present here two scenarios.

In the first simulation, Sky-Sailor starts a flight at EPFL location on the 21 th of June with an empty battery, keeping always the same altitude. The two graphs below show the evolution of the power distribution during 48 hours.

With good sun conditions, the battery is fully charged at $13 \mathrm{~h} 30$. At this moment, the MPPT measures that the battery voltage reaches the maximum 


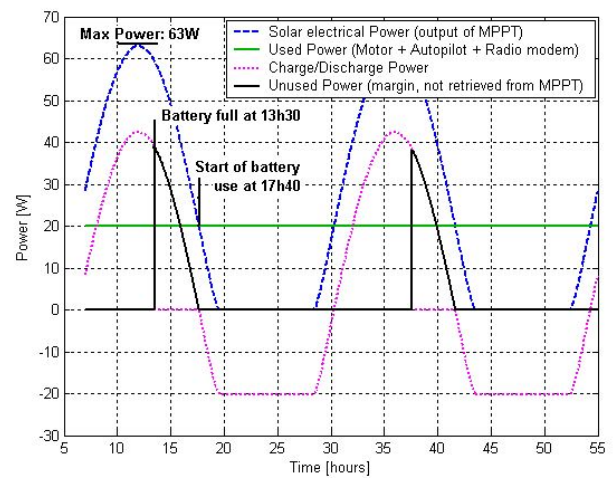

Fig. 7. Power distribution on Sky-Sailor during levelled flight

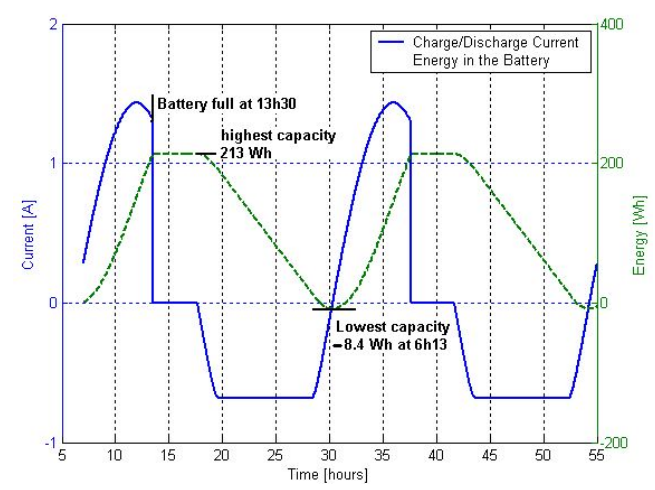

Fig. 8. Battery charge/discharge current and energy during levelled flight

voltage of $33.7[\mathrm{~V}]$ and adapts the maximum power point to avoid overcharge. In this phase, the total amount of energy that is not used but that could be retrieved from the solar panels reaches 92.5 [Wh]. During the night, the battery supplies the all airplane but at $5 \mathrm{~h} 10$ it is totally discharged.

Another strategy is to better use the energy after the battery charge by increasing altitude. Fig. 9, 10 and 11 show the same scenario presented before but with a climbing phase until 2000 [m].

Basically, Sky-Sailor uses the additional energy to gain altitude at 0.3 $[\mathrm{m} / \mathrm{s}]$ using an electrical power of $40[\mathrm{~W}]$. Having reached $2000[\mathrm{~m}]$, it stays at this altitude until the energy is not sufficient anymore for levelled flight. At this point, the motor is turned off and the descent starts. Finally, at the most critical point at $6 \mathrm{~h} 13$ in the next morning, the battery still has a capacity of $4.7[\mathrm{Wh}]$ and the charging process starts again. Globally, the unused energy during the day is 61.5 [Wh]. 


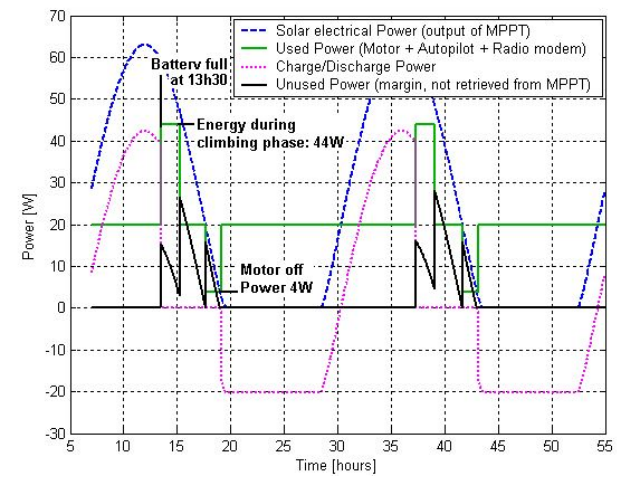

Fig. 9. Power distribution on Sky-Sailor during flight with climbing phase

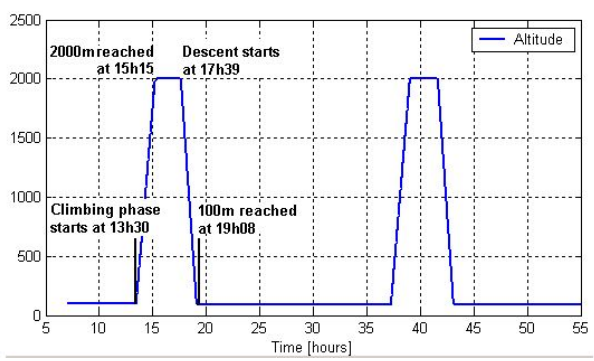

Fig. 10. Altitude during flight with climbing phase

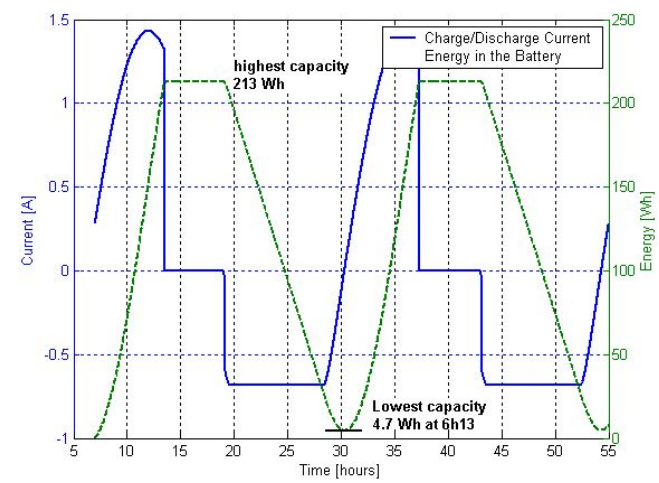

Fig. 11. Battery charge/discharge current and energy during flight with climbing phase 


\section{Status of the Project and Future Work}

The mechanical structure of the airplane is actually ready, it has been successfully tested and validated in terms of power and stability. The solar generator, composed of the solar modules and the MPPT, is in the integration phase on the wing.

Concerning the autopilot, the different parts of the system are being assembled and all functionalities will be tested during the first half of this year. In the summer, we should have achieved many flights and experiments to clearly evaluate the capabilities of our UAV.

\section{Potential Applications}

Small and high endurance UAVs find uses in a lot of varied fields, civilian or military. The civil applications, leaving side the military ones, could include coast or border surveillance, atmospherical and weather research and prediction, environmental, forestry, agricultural, and oceanic monitoring, imaging for the media and real-estate industries, and a lot of others. The target market for the following years is extremely important [11].

The great advantages of Sky-Sailor compared to other solutions would be without any doubt its capability to remain airborne for a very long period, its low cost and the simplicity with which it can be used and deployed, without any ground infrastructure for the lunch sequence.

As an example, in the hypothetical case of forest fire risks during a warm period, a dozen Sky-Sailor, easily launched with the hand, could efficiently monitor an extended surface, looking for fire starts. A fast report would allow a rapid intervention and thus reduce the cost of such disaster, in terms of human and material losses.

Sky-Sailor would be as well a very interesting platform for academic research, in aerodynamics or control.

\section{Conclusion}

In this paper, the design of an ultra-lightweight UAV was presented, including details about it's mechanical structure, the solar generator and the autopilot system. The approach adopted doesn't aim only at building an efficient autopilot, but also keeps in mind it's future application. This is done by designing and selecting all the parts to obtain a lightweight and low-power airplane. We plan to perform the first experiments with the autonomous airplane during the first half of this year and a long endurance flight this summer. 


\section{Acknowledgement}

The authors would like to thank all the people who contributed to the definition study, Samir Bouabdallah for fruitful discussions and advices on flying robots, Walter Engel for the realization of the mechanical structure and all the students who worked or are working on this project.

\section{References}

1. Davison A J (2003) Real-time simultaneous localization and mapping with a single camera, IEEE Int. Conf. on Computer Vision, ICCV-2003, pp. 1403-1410, Nice (France), October 2003

2. Lacroix S, Kung I K (2004) High resolution 3D terrain mapping with low altitude imagery, 8th ESA Workshop on Advanced Space Technologies for Robotics and Automation (ASTRA'2004), Noordwijk (Pays-Bas), 2-4 Novembre 2004

3. Eisenbeiss H (2004) A mini unmanned aerial vehicle (UAV): system overview and image acquisition, International Workshop on "Processing and visualization using high-resolution imagery" 18-20 November 2004, Pitsanulok, Thailand

4. Kim J.-H, Sukkarieh S (2002) Flight Test Results of GPS/INS Navigation Loop for an Autonomous Unmanned Aerial Vehicle (UAV), The 15th International Technical Meeting of the Satellite Division of the Institute of Navigation (ION) 24-27 September, 2002, Potland, OR, USA

5. Kim J.-H, Wishart S, Sukkarieh S (2003) Real-time Navigation, Guidance and Control of a UAV using Low-cost Sensors. In International Conference of Field and Service Robotics (FSR03), Japan, July 2003.

6. Brown A K, Lu Y (2004) Performance Test Results of an Integrated GPS/MEMS Inertial Navigation Package, Proceedings of ION GNSS 2004, Long Beach, CA, Sept. 2004

7. Atkins E M et al. (1998) Solus: An Autonomous Aircraft for Flight Control and Trajectory Planning Research, Proceedings of the American Control Conference, Pennsylvania, June 1998

8. Johnson E N et al. (2004) UAV Flight Test Programs at Georgia Tech, Proceedings of the AIAA Unmanned Unlimited Technical Conference, Workshop, and Exhibit, 2004.

9. Granlund G (2000) Witas: An intelligent autonomous aircraft using active vision. In Proceedings of the UAV 2000 International Technical Conference and Exhibition, Paris, France, June 2000. Euro UVS

10. DeGarmo M, Nelson G M (2004) Prospective Unmanned aerial vehicle operations in the future national airspace system, AIAA 4th Aviation Technology, Integration and Operations (ATIO) Forum, 20 - 23 Sept 2004, Chicago

11. Wong K.C, Bil C (1998) UAVs over Australia - Market And Capabilities, Paper No. 4, Proceedings of the 13th Bristol International Conference on RPVs/UAVs, Bristol, UK, 1998

12. Duffie J A, Beckman W A (1991) Solar Engineering of Thermal Processes, Second Edition. New York: Wiley-Interscience. 